\title{
Reconstruction in vitro de peau de patients atteints de xeroderma pigmentosum
}

Les ultraviolets (UV) contenus dans la lumière solaire, constituent la cause principale des cancers de la peau ( $30 \%$ de tous les cancers humains) [1]. Plus de $90 \%$ d'entre eux résultent de la transformation de kératinocytes de l'épiderme, cellules composant la couche superficielle de notre peau. Les UVB ( 290 et $320 \mathrm{~nm}$ ) provoquent dans I'ADN cellulaire des lésions localisées sur les séquences bipyrimidiques (enchaînements nucléotidiques de type CC, CT, TC, ou TT, $C$ : cytosine, $T$ : thymine). Pour assurer le maintien de l'intégrité du patrimoine génétique, ces lésions doivent être réparées, faute de quoi, elles peuvent conduire à l'introduction de mutations responsables du développement tumoral. Au moins $50 \%$ des tumeurs cutanées portent par exemple une mutation sur (au moins) le gène supresseur de tumeur p53.

Le mécanisme majeur de la réparation des lésions induites par les UVB dans I'ADN est «l'excision de nucléotides» (nucleotide excision repair, NER). Celle-ci procède en cinq étapes successives: reconnaissance de la lésion, déroulement de la double hélice $d^{\prime} A D N$, incision en $3^{\prime}$ puis en $5^{\prime}$ du brin d'ADN lésé, synthèse réplicative à partir du brin restant et ligature du brin néo-synthétisé. La saturation du système de réparation, ou son altération, favorise la persistance des lésions, la mutagenèse et le développement tumoral. II existe des maladies génétiques de la réparation de I'ADN, dont le xeroderma pigmentosum (XP) est le prototype. Le XP survient très rarement ( 1 individu sur 500000 environ est touché), et est transmis sur le mode autosomique et récessif (les parents hétérozygotes sont indemnes). Les patients XP présentent une sensibilité au soleil très éle- léré, et surtout, une prédisposition précoce (à partir de 4 à 5 ans) et exacerbée (environ 2000 fois supérieure à celle de la population normale) [2] aux tumeurs cutanées d'origine épidermique, au niveau des zones exposées. L'espérance de vie des patients $X P$ est très réduite ( 20 ans en moyenne). L'exérèse des tumeurs cutanées constitue l'essentiel des traitements proposés et la protection solaire reste encore le meilleur acte de prévention.

L'étude de la réparation de l'ADN dans les cellules hybrides somatiques obtenues par fusion de cellules provenant de différents malades XP a permis d'identifier sept groupes de complémentation XP (XP-A à XP-G). Chacun de ces groupes XP correspond à la mutation d'un gène $X P$ entraînant l'al tération spécifique d'une étape précoce de la NER. Les cellules de XP constituent donc un système génétique de choix pour mieux comprendre les processus précoces de la cancérogenèse et du vieillissement cutané. La plus grande difficulté est de pouvoir étudier ces mécanismes dans le contexte tissulaire. D'une part, il est en effet éthiquement exclu d'irradier les patients XP et d'étudier I'effet des UV sur leur peau. D'autre part, les «souris $X P$ », obtenues par génie génétique (le XP n'existe à l'état naturel que chez I'homme), nous confrontent à plusieurs limitations: leur(s) phénotype(s) diverge(nt) souvent de celui du modèle humain [3], la peau de souris diffère de la peau humaine par sa structure et les classes de tumeurs qu'elle est susceptible de développer, notamment après exposition aux UV; la réparation de l'ADN chez les rongeurs se fait uniquement de façon liée à la transcription.

La peau humaine est un organe com- plexe, schématiquement divisée en un compartiment support, le derme et un compartiment superficiel, l'épiderme. Le derme est surtout composé de macromolécules matricielles (collagènes, élastine, protéoglycanes) synthétisées par les fibroblastes de ce compartiment. L'épiderme est principalement composé de kératinocytes organisés en un épithélium pluristratifié et différencié dont le stade terminal représente la formation des couches cornées. A partir de ces deux types cellulaires, la peau peut être reconstruite in vitro [4]. Ce modèle tri-dimensionnel comprend un équivalent de derme et un épiderme différencié. Cet outil de choix constitue une alternative à l'approche in vivo pour l'étude des réponses aux UV [5].

Pour la première fois, l'amélioration des techniques préexistantes d'isolement et de culture des cellules XP nous a permis d'étudier dans un contexte tenant compte de l'architecture des compartiments cutanés, les caractéristiques de la peau malade reconstruite in vitro à partir de kératinocytes et de fibroblastes XP [6]. Cette réalisation a été possible grâce à l'étroite collaboration entre nos équipes de recherche à $L^{\prime} O$ réal et au Cnrs. L'étude a été focalisée sur le groupe de complémentation XP-C dont le phénotype est limité à la photosensibilité et à la prédisposition aux cancers cutanés, mais est exempt des troubles développementaux et neurologiques observés dans les autres groupes XP. Les cellules (kératinocytes et fibroblastes) des patients $X P$ ont été obtenues à partir de très petites biopsies de peau non exposée, puis soumises à des analyses moléculaires démontrant une déficience de réparation de l'ADN de 80 à $90 \%$, et prouvant, après complé- 
Figure 1. La peau xeroderma pigmentosum reconstruite in vitro. En $A$ et $B$, la coloration histologique de coupes de peau normale $(A)$ et de peau XP (B) reconstruites in vitro, révèle des différences de structure, notamment la présence d'une couche cornée (délimitée par les crochets blancs) plus fine dans l'épiderme XP (délimité par le crochet noir E), et la présence d'invaginations kératinocytaires (flèches rouges obliques) dans le derme équivalent XP (indiqué par le crochet noir D). En $C$ et $D$, les marquages immunohistochimiques ont révélé l'altération de certaines étapes de la différenciation kératinocytaire, notamment le retard et la diminution de l'expression de la kératine $\mathrm{K} 10$ dans l'épiderme XP illustrés ici. Les illustrations $E$ à $H$, indiquent que les lésions induites par les UVB et révélées à l'aide d'un anticorps spécifique (flèches rouges horizontales), sont totalement réparées dans l'épiderme normal (comparer $E$ et $G$ ), alors qu'elles persistent au moins jusqu'à 96 heures après irradiation dans l'épiderme XP (comparer $\mathrm{F}$ et $\mathrm{H}$ ).

mentation génétique, leur appartenance au groupe XP-C [7]. L'utilisation de fibroblastes et kératinocytes normaux et $X P$, provenant de quatre malades XP-C indépendants a permis de réaliser toutes les combinaisons possibles, derme normal et épiderme $X P$, derme $X P$ et épiderme normal, et «peau reconstruite $X P$ complète». Les résultats sont inattendus. Les kératinocytes XP ensemencés sur un derme contenant des fibroblastes normaux ont révélé l'altération du programme de différenciation épidermique par rapport aux kératinocytes normaux [8] (figure 1). Les épidermes XP ont montré un retard et une diminution dansl'expression des marqueurs de différenciation précoces (la kératine K10), et tardifs (la loricrine, la filaggrine). Au contraire, l'expression des chaînes d'intégrines $\alpha-6$ et $\beta-1$, des protéines impliquées dans l'ancrage des kératinocytes de l'assise basale et le contrôle de leur prolifération, a été significativement augmentée en parallèle à l'augmentation d'un facteur 10 environ de leur prolifération. Le petit nombre $\mathrm{m} / \mathrm{s} \mathrm{n}^{\circ} 12$, vol. 17, décembre 2001

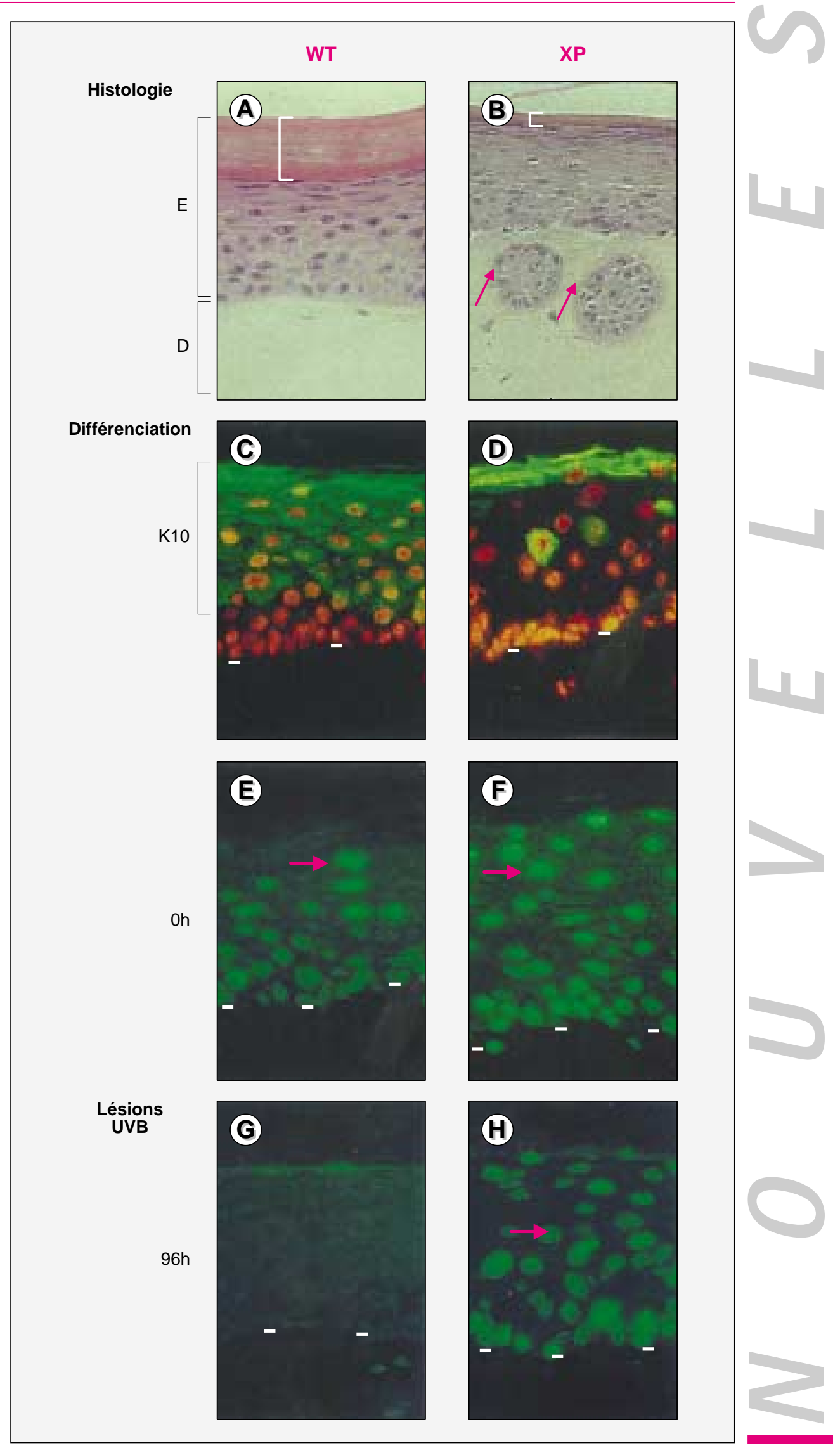


de contrôles, réalisables à partir de peaux XP ex vivo, a également révélé des altérations similaires de la différenciation épidermique.

$L$ 'incorporation de fibroblastes XP dans l'équivalent de derme a dévoilé leur influence, sur le comportement de kératinocytes normaux et, de façon encore plus marquée, quand ils sont XP. Ainsi, les kératinocytes $X P$ et normaux, cultivés sur équivalent de derme $X P$ ont montré une forte tendance à l'envahissement de la matrice, formant alors des figures troublantes d'invagination dont l'histologie rappelle les processus précoces de la cancérogenèse cutanée. Ces données suggèrent fortement que les fibroblastes X $P$ pourraient (peut être via l'expression de cytokines) avoir une influence prépondérante sur l'expression des métalloprotéases (MMPs), des enzymes capables de digérer et remanier la matrice extracellulaire. Cette hypothèse séduisante sera étudiée prochainement car certaines M MPs sont connues pour intervenir dans les processus d'invasion tumorale [9].

En dernier lieu, l'exposition des peaux XP reconstruites in vitro a clairement montré que les lésions induites par les UVB dans les noyaux des kératinocytes XP-C persistent au moins jusqu'à 96 heures après irradiation alors qu'elle sont éliminées en 24 heures dans la peau normale.

Trois conclusions majeures ressortent de l'étude. 1) Les capacités de différenciation des kératinocytes XP sont altérées. Ce phénomène, dont les causes moléculaires restent à élucider, pourrait contribuer à une moindre absorption des UV traversant l'épiderme, et donc affaiblir le «bouclier» protégeant normalement les kératinocytes de la couche basale épidermique, responsable du renouvellement du tissu, mais aussi à l'origine des tumeurs cutanées. En présence d'UV (y compris de faibles doses), cet affaiblissement pourrait aussi avoir des répercussions sur la matrice extracellulaire du derme, et expliquer en partie le vieillissement cutané précoce de la peau XP. 2) Les fibroblastes de derme $X P$ ont une influence considérable sur le comportement des kératinocytes de l'épi- remaniement de la matrice extracelIulaire, ce qui souligne l'importance des interactions mésenchyme-épithélium. 3) La déficience de la réparation des lésions à l'ADN induites par les UVB peut être facilement mise en évidence et étudiée dans la peau après reconstruction in vitro. La conjonction malheureuse des propriétés de différenciation et de prolifération de la peau $X P$, et de sa déficience en réparation de I'ADN, explique mieux pourquoi les UV «trouvent » dans la peau des malades un terrain de prédilection à l'induction du processus tumoral.

Les perspectives appliquées et fondamentales de la peau $X P$ reconstruite in vitro sont nombreuses. Une des plus évidentes et attendues est la thérapie cellulaire et tissulaire, engagée précédemment par la complémentation des fibroblastes XP-C [10]. Les progrès que nous avons accomplis depuis avec les kératinocytes XP-C indiquent que l'introduction du gène XPC sauvage dans ces cellules restaure leur capacité de réparer I'ADN et leur survie face aux UV ( résultats non publiés). La réversion des kératinocytes XP-C ne sera complète que si les capacités de différenciation et de prolifération sont, elles aussi, restaurées.

Grâce au développement de ce système organotypique hypersensible aux UV, des améliorations dans l'élaboration et le ciblage des traitements pharmacologiques destinés aux patients sont espérées. La peau XP reconstruite in vitro devrait permettre de mieux comprendre comment agissent les dérivés de la vitamine $A$, substances indispensables à I'homéostasie épidermique et très utilisées chez les patients XP où ils peuvent exercer une activité anti-tumorale partielle [11]. Enfin, la photosensibilité extrême de la peau XP suggère que celle-ci représente un outil majeur pour la mise au point de protections solaires plus efficaces.

Pour conclure, rappelons nous que la grande majorité des cancers cutanés résultent d'une exposition solaire immodérée et que ces cancers atteignent le taux impressionnant de $30 \%$ de la totalité des néoplasies humaines, valeur en augmentation constante depuis plusieurs décennies.
1. DePinho RA. The age of cancer. Nature 2000; 408: 248-54.

2. de Boer J, Hoeijmakers JH. Nucleotide excision repair and human syndromes. Carcinogen esis $2000 ; 21: 453-60$.

3. Friedberg EC. Cancer predisposition associated with defective DNA repair: studies with mutant mouse strains. Cancer J Sci Am 1999; 5: 257-63.

4. Asselineau D, Bernard B, Bailly C, Darmon M. Epidermal morphogenesis and induction of the $67 \mathrm{kD}$ keratin polypeptide by culture of human keratinocytes at the liquid-air interface. Exp Cell Res 1985; 159: 536-9.

5. Bernerd F, Asselineau D. Successive alteration and recovery of epidermal differentiation and morphogenesis after specific UVB-damages in skin reconstructed in vitro. Dev Biol 1997; 183: 123-38.

6. Bernerd F, Asselineau D, Vioux C, et al. Clues to epidermal cancer proneness revealed by reconstruction of DNA repair-deficient xeroderma pigmentosum skin in vitro. Proc Natl Acad Sci USA 2001; 98 : 7817-22.

7. Otto A, Riou L, Marionnet C, Mori T, Sarasin A, Magnaldo T. Differential behaviors toward utraviolet $A$ and $B$ radiations of fibroblasts and keratinocytes from normal and DNA-repair deficients individuals. Cancer Res 1999; 59: 1212-8.

8. Fuchs $E$, Byrne $C$. The epidermis: rising to the surface. Curr Opin Genet Dev 1994; 4: 725-36.

9. Curran S, Murray GI. Matrix metalloproteinases in tumour invasion and metastasis. J Pathol 1999; 189: 300-8.

10. Zeng $L$, Quilliet $X$, Chevallier-Lagente $O$, Eveno E, Sarasin A, Mezzina M. Retrovirus-mediated gene transfer corrects DNA repair defect of xeroderma pigmentosum cells of complementation groups A, B and C. Gene Ther 1997; 4: 107784.

11. Kraemer KH, DiGiovanna JJ, Peck G. Chemoprevention of skin cancer in xeroderma pigmentosum. J Dermatol 1992; 19: 715-8.

\section{Françoise Bernerd \\ Daniel Asselineau}

$L$ 'O réal Recherche, Centre de recherche $C$.Zviak, 90, rue du Général-Roguet, 92583 Clichy Cedex, France.

E-mail : fbernerd@recherche.loreal.com

\section{Alain Sarasin Thierry Magnaldo}

CNRS, UPR 2169 Instabilité gén étique et cancer, Institut AndréL woff, 7, rue GuyM ôquet, 94801 Villejuif, France. E-mail : magnaldo@infobiogen.fr 